\title{
Optical coherence tomography tissue coverage and characterization at six months after implantation of bioresorbable scaffolds versus conventional everolimus eluting stents in the ISAR-Absorb MI trial
}

\author{
Himanshu Rai ${ }^{1,6,7} \cdot$ Fernando Alfonso $^{2} \cdot$ Michael Maeng $^{3}$. Christian Bradaric ${ }^{4}$. Jens Wiebe ${ }^{1}$. Javier Cuesta ${ }^{2}$. \\ Evald Høj Christiansen ${ }^{3}$. Salvatore Cassese ${ }^{1}$. Petra Hoppmann ${ }^{4}$. Roisin Colleran ${ }^{1,6,7} \cdot$ Fiona Harzer $^{1} \cdot$ Jola Bresha $^{1}$. \\ Nejva Nano ${ }^{1}$. Simon Schneider ${ }^{4}$ Karl-Ludwig Laugwitz ${ }^{4,5} \cdot$ Michael Joner $^{1,5} \cdot$ Adnan Kastrati $^{1,5} \cdot$ Robert A. Byrne $^{6,7}$
}

Received: 1 December 2020 / Accepted: 16 April 2021 / Published online: 21 August 2021

(c) The Author(s) 2021

\begin{abstract}
Purpose Data regarding vessel healing by optical coherence tomography (OCT) after everolimus-eluting bioresorbable scaffolds (BRS) or everolimus-eluting metallic stent (EES) implantation in acute myocardial infarction (AMI) patients is scarce. We compared OCT findings after BRS or EES implantation in patients with AMI enrolled in a randomized trial. Methods In ISAR-Absorb MI, AMI patients were randomized to BRS or EES implantation, with 6-8 month angiographic follow-up. This analysis includes patients who underwent OCT during surveillance angiography. Tissue characterization was done using grey-scale signal intensity analysis. The association between OCT findings and target lesion failure (TLF) at 2 years was investigated.

Results OCT was analyzed in 103 patients (2237 frames, 19,827 struts) at a median of 216 days post-implantation. Of these, 70 were treated with BRS versus 32 with EES. Pre-(92.8 vs. $68.7 \%, \mathrm{p}=0.002)$ and post-dilation $(51.4$ vs. $12.5 \%, \mathrm{p}<0.001)$ were more common in BRS as compared to EES. Strut coverage was higher in BRS vs. EES $(97.5 \%$ vs. $90.9 \%$, p < 0.001$)$. Mean neointimal thickness was comparable in both groups $[85.5(61.9,124.1)$ vs. $69.5(32.7,127.5) \mu \mathrm{m}$, respectively, $\mathrm{p}=0.20]$. Mature neointimal regions were numerically more common in BRS ( $43.0 \%$ vs. $24.6 \% ; \mathrm{p}=0.35)$; this difference was statistically significant in ST-elevation myocardial infarction patients ( $40.9 \%$ vs. $21.1 \%, \mathrm{p}=0.03)$.

At two-years, 8 (7.8\%) patients experienced TLF. Mean neointimal area $\left[0.61(0.21,1.33)\right.$ vs. $\left.0.41(0.11,0.75) \mathrm{mm}^{2}, \mathrm{p}=0.03\right]$ and mean neointimal coverage $[106.1(65.2,214.8)$ vs. $80.5(53.5,122.1) \mu \mathrm{m}, \mathrm{p}<0.01]$ were higher, with comparable tissue maturity, in lesions with versus without TLF.

Conclusions In selected patients who underwent OCT surveillance 6-8 months after coronary intervention for AMI with differing implantation characteristics depending on the device type used, vessel healing was more advanced in BRS compared with EES, particularly in the STEMI subgroup.
\end{abstract}

Keywords Bioresorbable scaffold · Acute myocardial infarction · Malapposition · Optical coherence tomography · Greyscale signal intensity $\cdot$ Uncovered struts

Robert A. Byrne

robebyrne@gmail.com

1 Deutsches Herzzentrum München, Technische Universität München, Munich, Germany

2 Hospital Universitario de La Princesa Madrid, Madrid, Spain

3 Aarhus University Hospital, Aarhus, Denmark

4 Medizinische Klinik Und Poliklinik Innere Medizin I, Klinikum Rechts Der Isar, Technische Universität München, Munich, Germany
5 DZHK (German Centre for Cardiovascular Research), Partner Site Munich Heart Alliance, Munich, Germany

6 Cardiovascular Research Institute Dublin, Mater Private Network, Dublin, Ireland

7 School of Pharmacy and Biomolecular Sciences, RCSI University of Medicine and Health Sciences, Dublin, Ireland 


\section{Abbreviations}

AMI Acute myocardial infarction

BRS Bioresorbable-scaffold

EES Everolimus-eluting stents

GSI Grey-scale signal intensity

OCT Optical coherence tomography

PCI Percutaneous coronary intervention

QCA Quantitative coronary angiography

ROI Region of interest

\section{Introduction}

Everolimus-eluting bioresorbable scaffolds (BRS) were designed with the intention of overcoming the long-term limitations of conventional metallic drug eluting stents (DES) [1]. The technology aims to provide temporary mechanical scaffolding with anti-proliferative drug release in the early period after implantation, with slow resorption thereafter to eliminate any nidus for late stent failure as seen with DES, caused by restenosis or stent thrombosis. It was hypothesized that complete resorption would facilitate return of vasomotor function and expansive remodeling of the treated arterial segment late after implantation.

Randomized clinical trials have shown significantly higher rates of target lesion failure and device thrombosis with the everolimus-eluting BRS as compared to conventional stents at mid- and long-term follow-up [2, 3], However, one area where BRS may offer an advantage over metallic stents is the setting of ST-elevation myocardial infarction (STEMI). Two trials have compared BRS and conventional EES exclusively in patients with acute myocardial infarction (AMI) [4, 5] The ISAR-Absorb MI trial showed comparable angiographic outcomes after BRS- or EES-implantation at 6-8 months. The ABSORB STEMITROFI II trial showed a lower healing score (as assessed by OCT) within BRS compared with EES at 6 months. Although neither trial was adequately powered to assess clinical outcomes, a meta-analysis of individual patient data from these two trials showed comparable clinical outcomes in the BRS and EES groups at follow-up, although this analysis was also impacted by limited power [2].

The present study involves a subset of subjects enrolled in the ISAR-Absorb MI trial who underwent optical coherence tomography (OCT) surveillance at the time of angiographic follow-up. Our primary objective was to investigate differences in the vessel healing processes 6-8 months after BRS- versus EES-implantation as assessed by OCT. Our secondary objective was to identify OCT factors associated with subsequent target lesion failure (TLF) out to 2 years post-stenting.

\section{Materials and methods}

\section{Patient population}

This study includes the subset of patients enrolled in the Intracoronary $\underline{\text { Scaffold }} \underline{\text { Assessment a }} \underline{\text { Randomized evalu- }}$

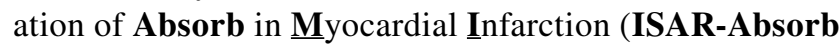
MI) Trial who underwent OCT surveillance at the time of routine angiographic follow-up at 6-8 months. OCT surveillance at the time of angiographic follow-up was not protocol-mandated and was done at the operator's discretion. Subjects who underwent target lesion revascularization before or at 6-8 month angiographic follow-up were excluded.

ISAR-Absorb MI was an investigator-initiated, prospective, randomized, multicenter, non-inferiority, clinical trial with a 2:1 treatment allocation to everolimus-eluting BRS (Absorb; Abbott Vascular, Santa Clara, CA, USA) vs. durable polymer everolimus-eluting stents (EES) in patients undergoing percutaneous coronary intervention for AMI (ClinicalTrials.gov Identifier: NCT01942070). Details of the trial design and primary results have been published elsewhere [5]. In brief, patients $>18$ years presenting with STEMI or NSTEMI, if accompanied by visual evidence of thrombosis on angiography, with planned stent implantation in a de novo lesion in a native vessel or coronary bypass graft with a reference vessel diameter of $\geq 2.5 \mathrm{~mm}$ and $\leq 3.9 \mathrm{~mm}$ were included. Key exclusion criteria included target lesions located in the left main coronary artery, severely calcified lesions, bifurcation lesions with a side branch diameter $>2 \mathrm{~mm}$, and any comorbid conditions with a life expectancy $<1$ year or that might result in protocol non-compliance. OCT surveillance at 6-8 month angiographic follow-up was done at the investigator's discretion. At three of the five participating centers, OCT surveillance was done routinely as part of clinical practice. All images obtained were sent to a centralized core laboratory [Intracoronary Stenting and Antithrombotic Research (ISAR) Center, Deutsches Herzzentrum München, Munich, Germany] as raw data for off-line analyses. Angiographic sequences and OCT pullbacks were measured by independent readers experienced in quantitative coronary angiography (QCA) and OCT analysis. Patients underwent additional clinical follow-up at two years post-procedure (16-18 months after angiographic/OCT surveillance) during which TLF incidence was recorded. TLF was defined as a composite of cardiac death, target-vessel myocardial infarction and target lesion revascularization. 


\section{QCA analysis}

QAngio XA 7.3.96.0 (Medis Medical Imaging Systems, Leiden, NL) was used for QCA analysis. Measurements were performed on cineangiograms recorded after the administration of intracoronary nitro-glycerine using the same single worst-view projection at all times. The contrast-filled non-tapered catheter tip was used for calibration before quantitative analysis. Both "in-stent" (stented segment) and "in-segment" (5-mm margins proximal and distal to the stent) areas were analyzed. Late lumen loss was defined as the difference between the minimal luminal diameter at the end of the procedure and the minimal luminal diameter at follow-up angiography. Binary angiographic restenosis was defined as diameter stenosis of $>50 \%$ in the in-segment area at follow-up. Standard criteria were used for determining qualitative morphological lesion characteristics.

\section{OCT analysis}

OCT pullbacks were acquired using a standard non-occlusive technique with a Frequency Domain-OCT intravascular imaging system (Abbott Laboratories, Illinois, USA) and a Dragonfly $^{\mathrm{TM}}$ DUO $^{\mathrm{TM}}$ or OPTIS ${ }^{\mathrm{TM}}$ catheter (Abbott Laboratories, Illinois, USA). Unitary image acquisition length was $75 \mathrm{~mm}$ or $54 \mathrm{~mm}$, while the pullback acquisition rate was 36 or $18 \mathrm{~mm} / \mathrm{sec}$ respectively.

QIvus 3.0.30.0 software (Medis Medical Imaging Systems, Leiden NL) was used to perform morphometric analysis as per standard operating procedure of the OCT corelab. Contiguous cross-sections within the stented segment spaced at each $1 \mathrm{~mm}$ longitudinal interval were analyzed. Parameters assessed for morphometric analysis included stent area, lumen area, stent diameter, neointimal area, neointimal thickness and percentage area of stenosis [6]. Stent/scaffold expansion index was calculated according to the criteria using the formula: minimum stent or scaffold area/reference lumen area [7, 8]. Struts were adjudicated as covered when the neointimal tissue overlying each stent strut was $\geq 20 \mu \mathrm{m}$ (minimum axial resolution of OCT) and uncovered if the neointimal tissue overlying each strut was $<20$ or $<30 \mu \mathrm{m}$ (for EES and BRS, respectively) [9, 10]. We assumed some degree of irregular degradation of poly-d,llactide coating along with the poly-L-lactic acid strut backbone at 6-8 month post-implant follow-up. Accordingly, we regarded the minimum distance from the strut surface to the lumen contour (in the direction of the gravitational center of the vessel) as neointimal thickness overlying that strut. Struts were adjudicated as malapposed when strut center-tolumen contour distance was more than: stent/scaffold strut thickness + polymer thickness + minimal axial resolution of OCT. Struts located at the ostium of side branches, with no vessel wall behind, were designated as non-apposed sidebranch (NASB) struts and were excluded from the apposition analysis.

\section{GSI analysis}

Neointimal tissue characterization as mature or immature was performed on OCT cross sections using offline GSI analysis. OCT images were manually transferred to an image editing software (ImageJ, Version $1.48 \mathrm{~g}, 2013$ ) at a resolution of $1024 \times 1024$ pixel and converted to a grey scale signal (8-bit) as preparation for GSI analysis. Contiguous cross-sections within the stented segment spaced at $1 \mathrm{~mm}$ longitudinal intervals were analyzed. The neointimal region of interest (ROI) above each covered stent strut was delineated and 256-level GSI was measured for every pixel within the ROI. GSI analysis was performed only for neointimal ROIs with a thickness of 100 to $400 \mu \mathrm{m}$. Tissue coverage was classified as mature or immature according to a standard cut-off GSI score of 109.7 based on a prior pre-clinical study and a pilot clinical investigation [11].

\section{Statistical analysis}

Continuous data are presented as mean $\pm \mathrm{SD}$ or median (Interquartile range, IQR). Categorical data are presented as observed frequencies and proportions (\%). Morphometric OCT parameters and GSI results were compared between the two stent groups (BRS vs. EES) for the whole cohort and for the STEMI subgroup. Categorical variables were compared between the two stent groups using the chi ${ }^{2}$ test with Yates' continuity correction or the Fisher's exact test (where at least one expected cell value was expected to be $<5$ ). Continuous variables were compared using Student's t-test or Wilcoxon rank sum test, as appropriate. Generalized linear mixed models (GLMM) were used as appropriate to account for cluster variability. Inter-and intra-observer variability for strut coverage, apposition and GSI-aided tissue characterization was evaluated using Cohen's Kappa ( $\kappa$ ) coefficient in $\sim 5 \%$ of frames, which were randomly selected. A twosided $P$-value $\leq 0.05$ was considered as an indicator of statistical significance. All statistical analyses were performed using R (version 3.5.0, R Core Team, R Foundation, Vienna, Austria).

\section{Results}

A total of 119 cases enrolled in ISAR-Absorb-MI had OCT imaging performed at the time of 6-8 month angiographic follow-up. Of these, 17 failed the selection process for morphometric analysis. The remaining 102 cases were suitable for morphometric analysis (70 BRS cases; 32 EES 
Fig. 1 Study flow chart

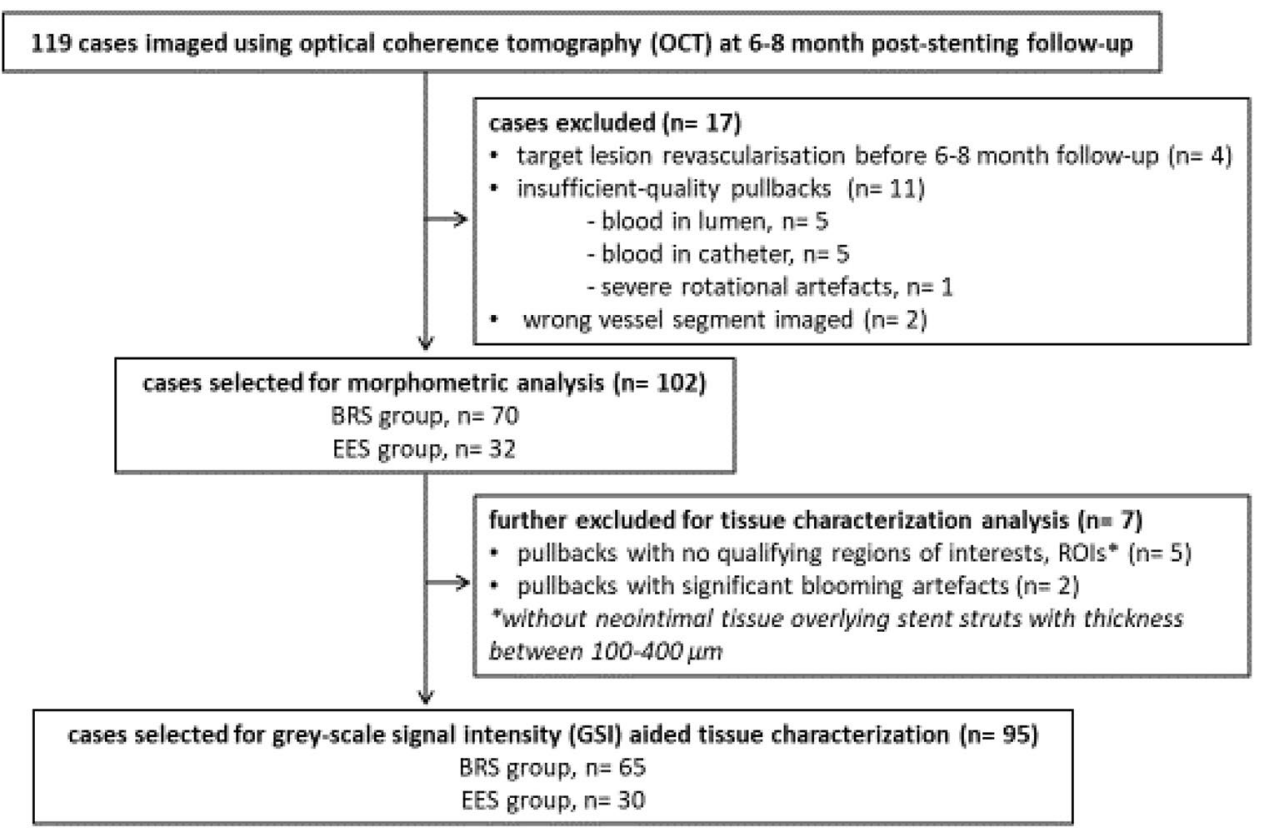

cases); of these 95 cases were suitable for GSI analysis (65 BRS cases; 30 EES cases) (Fig. 1).

There were differences observed between the baseline and procedural characteristics of patients with and without
OCT follow-up suitable for morphometric analysis (see Supplementary Table 1 and Supplementary Table 2).

Baseline patient characteristics are shown in Table 1. Patients in the BRS group as compared to EES group were younger $(59.3 \pm 9.5$ vs. $64.3 \pm 11.2$ years, respectively,
Table 1 Baseline patient characteristics

\begin{tabular}{llll}
\hline & BRS & EES & P-value \\
\hline Patients & 70 & 32 & \\
Age, years & $59.3 \pm 9.5$ & $64.3 \pm 11.2$ & 0.03 \\
Female gender & $7(10.0)$ & $7(21.9)$ & 0.11 \\
Diabetes mellitus & $14(20.0)$ & $5(15.6)$ & 0.60 \\
Insulin dependent & $2(2.9)$ & $0(0)$ & 0.33 \\
Hypertension & $31(44.3)$ & $13(40.6)$ & 0.73 \\
Current smoking & $37(52.9)$ & $17(53.1)$ & 0.98 \\
Family history of CAD & $10(14.3)$ & $2(6.2)$ & 0.30 \\
Prior percutaneous coronary intervention & $4(5.7)$ & $1(3.1)$ & 0.67 \\
Prior myocardial infarction & $4(5.7)$ & $0(0)$ & 0.17 \\
Number of vessels diseased & & & 0.15 \\
1 Vessel disease & $53(75.7)$ & $19(59.4)$ & \\
2 Vessel disease & $12(17.1)$ & $7(21.9)$ & \\
3 Vessel disease & $5(7.1)$ & $6(18.7)$ & 0.19 \\
Clinical presentation & & & \\
ST-elevation myocardial infarction & $60(85.7)$ & $24(75.0)$ & $8(25.0)$ \\
Non-ST-elevation myocardial infarction & $10(14.3)$ & & 0.25 \\
STEMI location/presentation & & $10(41.7)$ & $1(4.2)$ \\
Anterior & $31(51.7)$ & $4.44 \pm 4.65$ & \\
Lateral & $9(15.0)$ & & \\
Posterior & $20(33.3)$ & $6.09 \pm 9.56$ & \\
Troponin (max), ng/dl & & & \\
\hline & & &
\end{tabular}

Data shown as mean \pm SD or number (percentage) 
Table 2 Baseline lesion and angiographic characteristics

\begin{tabular}{|c|c|c|c|}
\hline & BRS & EES & P-value \\
\hline Lesions & 70 & 32 & \\
\hline Target vessel & & & 0.18 \\
\hline Left anterior descending & $34(48.6)$ & $14(43.7)$ & \\
\hline Left circumflex & $12(17.1)$ & $2(6.2)$ & \\
\hline Right coronary artery & $24(34.3)$ & $16(50.0)$ & \\
\hline Bifurcation & $15(21.7)$ & $6(18.7)$ & 0.73 \\
\hline Pre-dilation & $64(92.8)$ & $22(68.7)$ & 0.002 \\
\hline Stent diameter, $\max (\mathrm{mm})$ & $3.1 \pm 0.4$ & $3.1 \pm 0.4$ & 0.96 \\
\hline Total stented length (mm) & $21.4 \pm 9.6$ & $22.8 \pm 10.7$ & 0.54 \\
\hline Nominal diameter of largest balloon (mm) & $3.2 \pm 0.4$ & $3.2 \pm 0.4$ & 0.51 \\
\hline Balloon pressure, $\max (\mathrm{atm})$ & $17.7 \pm 3.2$ & $16.6 \pm 3.1$ & 0.10 \\
\hline Post-dilation & $36(51.4)$ & $4(12.5)$ & $<0.001$ \\
\hline TIMI flow, post PCI & & & 0.28 \\
\hline 0 & $0(0)$ & $1(3.1)$ & \\
\hline 1 & $0(0)$ & $0(0)$ & \\
\hline 2 & $1(1.4)$ & $1(3.1)$ & \\
\hline 3 & $69(98.6)$ & $30(93.7)$ & \\
\hline \multicolumn{4}{|l|}{ Quantitative coronary angiography analysis } \\
\hline \multicolumn{4}{|l|}{ Pre-intervention } \\
\hline Reference diameter (mm) & $2.85 \pm 0.39$ & $2.88 \pm 0.37$ & 0.71 \\
\hline Minimal lumen diameter (mm) & $0.30 \pm 0.36$ & $0.16 \pm 0.34$ & 0.06 \\
\hline Diameter stenosis $(\%)$ & $89.6 \pm 12.7$ & $94.8 \pm 11.2$ & 0.04 \\
\hline \multicolumn{4}{|l|}{ Post-intervention } \\
\hline Reference diameter (mm) & $2.95 \pm 0.39$ & $2.98 \pm 0.37$ & 0.65 \\
\hline Minimal lumen diameter, in-stent (mm) & $2.59 \pm 0.36$ & $2.69 \pm 0.37$ & 0.24 \\
\hline Minimal lumen diameter, in-segment (mm) & $2.31 \pm 0.45$ & $2.26 \pm 0.44$ & 0.66 \\
\hline Diameter stenosis, in-stent (\%) & $11.8 \pm 5.9$ & $9.9 \pm 5.6$ & 0.13 \\
\hline Diameter stenosis, in-segment (\%) & $22.0 \pm 9.3$ & $24.1 \pm 12.7$ & 0.41 \\
\hline
\end{tabular}

Data shown as mean \pm SD or number (percentage) $\mathrm{p}=0.03$ ) but the groups were otherwise well matched. Lesion and procedural characteristics are shown in Table 2. Pre-dilation $(92.8 \%$ vs. $68.7 \%, \mathrm{p}=0.002)$ and post-dilation $(51.4 \%$ vs. $12.5 \%, \mathrm{p}<0.001)$ were more frequently done in patients treated with BRS. Lesion and procedural characteristics were otherwise well matched between the two groups.

Angiographic and OCT surveillance of patients in the present study was performed at a median follow-up was 216 days post-intervention. QCA results of follow-up
Table 3 Angiographic follow-up at 6-8 months

\begin{tabular}{llll}
\hline & BRS & EES & P-value \\
\hline Lesions & 70 & 32 & \\
Days to angiographic follow-up & $216[204,233]$ & $215[205,239]$ & 0.70 \\
Reference diameter, mm & $2.90 \pm 0.43$ & $2.97 \pm 0.38$ & 0.46 \\
Minimal lumen diameter, in-stent (mm) & $2.38 \pm 0.47$ & $2.51 \pm 0.53$ & 0.23 \\
Minimal lumen diameter, in-segment (mm) & $2.18 \pm 0.50$ & $2.21 \pm 0.45$ & 0.78 \\
Diameter stenosis, in-stent (\%) & $18.0 \pm 11.6$ & $15.7 \pm 12.3$ & 0.39 \\
Diameter stenosis, in-segment (\%) & $25.0 \pm 12.4$ & $25.6 \pm 11.1$ & 0.79 \\
Late lumen loss, in-stent (mm) & $0.21 \pm 0.29$ & $0.17 \pm 0.36$ & 0.60 \\
Late lumen loss, in-segment (mm) & $0.12 \pm 0.38$ & $0.05 \pm 0.45$ & 0.47 \\
Binary restenosis & $5(7.1)$ & $1(3.1)$ & 0.42 \\
\hline
\end{tabular}

Data shown as mean $\pm \mathrm{SD}$ or median $[\mathrm{IQR}]$ or number (percentage) 
Fig. 2 Principal angiographic and OCT findings at 6-8-month follow-up. a Comparison of in-segment percentage diameter stenosis between BRS and EES; b Proportion of uncovered struts at 6-8 months follow-up between BRS and EES
A In-segment diameter stenosis at follow-up, \%

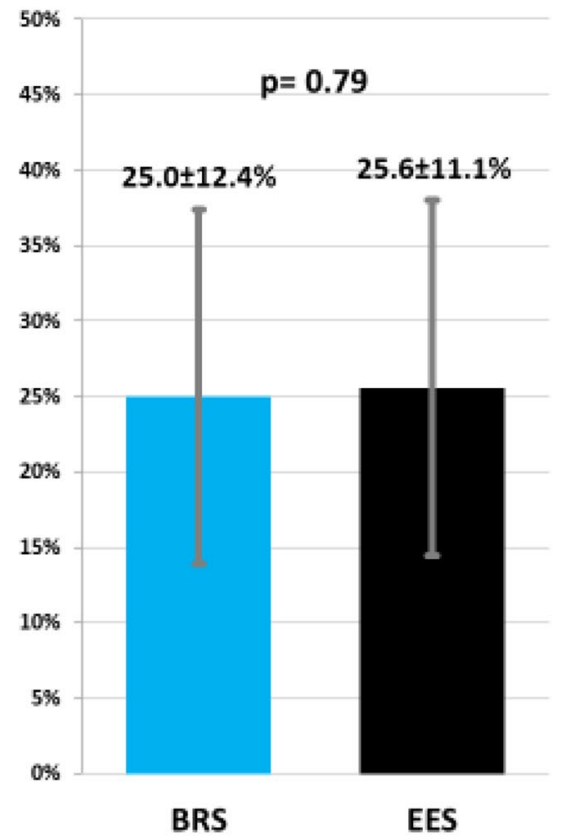

B Uncovered struts at follow-up, \%

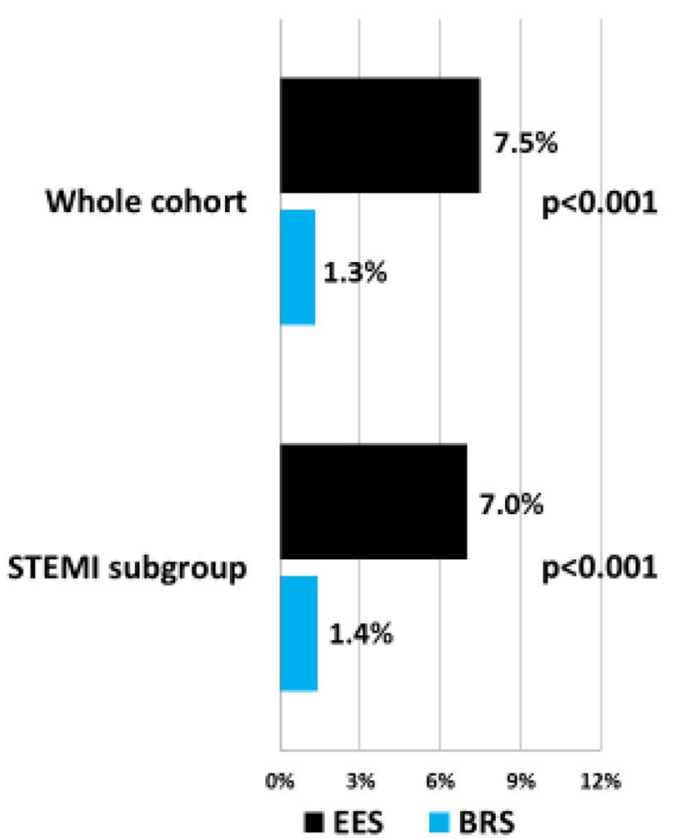

angiogram are shown in Table 3. In-segment diameter stenosis, which was the primary endpoint measure in the main study, was comparable between BRS and EES $(25.0 \pm 12.4$ vs. $25.6 \pm 11.1 \%, p=0.79$ ). (Fig. 2, Panel A) In-stent and in-segment minimal lumen diameters and late lumen loss were also comparable between the two treatment groups.

OCT analysis results are shown in Table 4. In total, 2237 frames with 19,827 struts were assessed. Minimum lumen area $\left[5.13(3.95,6.70)\right.$ vs. $\left.4.93(3.84,6.99) \mathrm{mm}^{2}\right]$ and minimum stent area $[5.78(4.88,7.34)$ vs. $6.39(4.77$, 7.45) $\mathrm{mm}^{2}$ ] were comparable between BRS and EES in the whole cohort, as well as in the STEMI subgroup. Stent/ scaffold expansion index was marginally lower in BRS as compared to EES in the whole cohort $[0.75(0.64,0.83)$ vs. $0.81(0.71,0.90), \mathrm{p}=0.05]$, and significantly lower in BRS in the STEMI subgroup $[0.74(0.64,0.82)$ vs. $0.81(0.71$, 0.90), $\mathrm{p}=0.01$ ] (Fig. 3).

Amongst frame level measurements, neointimal area [0.41 $(0.10,0.77)$ vs. $\left.0.43(0.15,0.77) \mathrm{mm}^{2}, \mathrm{p}=0.73\right]$ and percentage area stenosis $[6.15(1.27,11.22)$ vs. $5.95(2.06$, 12.16) $\%, p=0.77]$ as well as rest of the parameters were comparable between BRS and EES in the whole cohort.

The percentage of uncovered struts was significantly less common in BRS as compared to EES in the whole cohort $(1.3 \%$ vs. $7.5 \%, \mathrm{p}<0.001)$, as well as in the STEMI subgroup (1.4\% vs. $7.0 \%, \mathrm{p}<0.001$ ) (Fig. 2, Panel B). Amongst other strut-level measurements, strut coverage was found to better with BRS compared to EES $(97.5 \%$ vs. $90.9 \%, \mathrm{p}<0.001)$. Malapposed struts were numerically less common with BRS (0.5\% vs. $1.1 \%, \mathrm{p}=0.51)$. Neointimal thickness was also numerically higher in BRS as compared to EES $[69.5(32.7,127.5)$ vs. $85.5(61.9,124.1) \mu \mathrm{m}$, $\mathrm{p}=0.20]$. Results were consistent for each parameter in the STEMI subgroup. Inter-and intra-observer variability for strut apposition assessed randomly in 122 frames (1072 struts) showed high concordance $(\kappa=0.91$ and $\kappa=0.93$, respectively).

Results of tissue characterization by GSI analysis are summarized in Table 5. Mature ROIs were numerically more common in BRS compared to EES (43.0\% vs. $24.6 \%$; $\mathrm{p}=0.35)$; this difference was statistically significant in STEMI subgroup (40.9\% vs. 21.1\%, p=0.03) (Fig. 4). Inter-and intra-observer variability for neointimal tissue characterization assessed in 117 frames (219 ROIs) also showed high concordance $(\kappa=0.90$ and $\kappa=0.90$, respectively).

Strut-lumen distances obtained for all analyzed struts in both EES and BRS groups are visually represented as Fig. 5.

TLF occurred in $8(7.8 \%)$ patients in the interval between 6 and 8 month post PCI OCT surveillance and 2 years clinical follow-up. Mean neointimal area $[0.61(0.21,1.33)$ vs. $\left.0.41(0.11,0.75) \mathrm{mm}^{2}, \mathrm{p}=0.03\right]$ and mean neointimal thickness above struts $[106.1(65.2,214.8)$ vs. $80.5(53.5,122.1)$ $\mu \mathrm{m}, \mathrm{p}<0.01]$ were higher in patients with TLF. Patients with and without subsequent TLF had comparable GSI scores and proportions of mature ROIs ( $\mathrm{p}=0.96$ and 0.39 , respectively) (Data not shown). 
Table 4 Results from morphometric OCT analysis

\begin{tabular}{|c|c|c|c|c|c|c|}
\hline & \multicolumn{3}{|l|}{ Whole cohort } & \multicolumn{3}{|l|}{ STEMI subgroup } \\
\hline & BRS & EES & $P$-value & BRS & EES & $P$-value \\
\hline \multicolumn{7}{|l|}{ Patient-level measurements } \\
\hline Patients, $n$ & 70 & 32 & & 60 & 24 & \\
\hline Stented length, mm & $19.8(13.5,24.5)$ & $21.7(16.6,26.6)$ & 0.77 & $19.25(13.47,23.85)$ & $21.7(16.92,27.5)$ & 0.53 \\
\hline Reference lumen diameter, mm & $3.25(2.81,3.70)$ & $3.11(2.73,3.46)$ & 0.31 & $3.3(2.83,3.77)$ & $2.98(2.73,3.36)$ & 0.09 \\
\hline Reference lumen area, $\mathrm{mm}^{2}$ & $8.38(6.33,10.88)$ & $7.65(5.80,9.44)$ & 0.22 & $8.60(6.35,11.19)$ & $7.02(5.80,8.86)$ & 0.04 \\
\hline Minimum lumen diameter, $\mathrm{mm}$ & $2.56(2.24,2.92)$ & $2.50(2.21,2.98)$ & 0.97 & $2.53(2.24,2.85)$ & $2.47(2.21,2.82)$ & 0.58 \\
\hline Maximum lumen diameter, $\mathrm{mm}$ & $3.26(2.92,3.86)$ & $3.28(2.87,3.75)$ & 0.64 & $3.22(2.92,3.80)$ & $3.2(2.87,3.73)$ & 0.40 \\
\hline Minimum lumen area, $\mathrm{mm}^{2}$ & $5.13(3.95,6.70)$ & $4.93(3.84,6.99)$ & 0.96 & $5.04(3.94,6.84)$ & $4.79(3.84,6.26)$ & 0.49 \\
\hline Maximum lumen area, $\mathrm{mm}^{2}$ & $8.35(6.70,11.71)$ & $8.49(6.46,11.03)$ & 0.59 & $8.17(6.67,11.34)$ & $8.05(6.46,10.95)$ & 0.32 \\
\hline Minimum stent diameter, mm & $2.71(2.47,3.06)$ & $2.88(2.46,3.09)$ & 0.52 & $2.69(2.46,3.14)$ & $2.88(2.46,3.09)$ & 0.65 \\
\hline Maximum stent diameter, $\mathrm{mm}$ & $3.29(2.90,3.78)$ & $3.29(2.89,3.59)$ & 0.40 & $3.27(2.89,3.67)$ & $3.26(2.89,3.59)$ & 0.50 \\
\hline Minimum stent area, $\mathrm{mm}^{2}$ & $5.78(4.88,7.34)$ & $6.39(4.77,7.45)$ & 0.73 & $5.70(4.83,7.72)$ & $6.19(4.77,7.32)$ & 0.95 \\
\hline Maximum stent area, $\mathrm{mm}^{2}$ & $8.52(6.67,11.25)$ & $8.50(6.53,10.14)$ & 0.30 & $8.41(6.64,10.57)$ & $8.36(6.53,10.14)$ & 0.37 \\
\hline Minimum neointimal area, $\mathrm{mm}^{2}$ & $-0.41(-1.02,-0.01)$ & $-0.23(-0.63,0.09)$ & 0.78 & $-0.415(-1.05,0.01)$ & $-0.20(-0.63,0.14)$ & 0.24 \\
\hline Maximum neointimal area, $\mathrm{mm}^{2}$ & $1.09(0.71,1.75)$ & $1.30(0.73,1.57)$ & 0.49 & $1.01(0.70,1.49)$ & $1.35(0.98,1.70)$ & 0.16 \\
\hline Stent/scaffold expansion index & $0.75(0.64,0.83)$ & $0.81(0.71,0.90)$ & 0.05 & $0.74(0.64,0.82)$ & $0.81(0.71,0.90)$ & 0.01 \\
\hline \multicolumn{7}{|l|}{ Frame-level measurements } \\
\hline Assessed frames, $\mathrm{n}$ & 1,529 & 708 & & 1,268 & 528 & \\
\hline Lumen diameter, mm & $2.91(2.58,3.34)$ & $2.94(2.56,3.31)$ & 0.84 & $2.88(2.58,3.35)$ & $2.83(2.54,3.23)$ & 0.61 \\
\hline Lumen area, $\mathrm{mm}^{2}$ & $6.64(5.23,8.74)$ & $6.79(5.15,8.63)$ & 0.76 & $6.52(5.22,8.83)$ & $6.30(5.07,8.21)$ & 0.53 \\
\hline Stent diameter, mm & $3.02(2.69,3.42)$ & $3.10(2.70,3.40)$ & 0.89 & $2.99(2.68,3.42)$ & $3.04(2.67,3.36)$ & 0.83 \\
\hline Stent area, $\mathrm{mm}^{2}$ & $7.13(5.69,9.16)$ & $7.57(5.73,9.06)$ & 0.83 & $7.00(5.64,9.19)$ & $7.26(5.61,8.86)$ & 0.77 \\
\hline Neointimal area, $\mathrm{mm}^{2}$ & $0.41(0.10,0.77)$ & $0.43(0.15,0.77)$ & 0.73 & $0.40(0.09,0.72)$ & $0.49(0.19,0.86)$ & 0.17 \\
\hline Percentage area stenosis, $\%$ & $6.15(1.27,11.22)$ & $5.95(2.06,12.16)$ & 0.77 & $5.75(1.18,10.84)$ & $7.43(2.43,13.13)$ & 0.20 \\
\hline \multicolumn{7}{|l|}{ Strut-level measurements } \\
\hline Visible struts, $n$ & 12,704 & 7,123 & & 10,527 & 5,236 & \\
\hline Covered struts, $\%$ & 97.5 & 90.9 & $<0.001$ & 97.4 & 91.5 & $<0.001$ \\
\hline Uncovered struts, $\%$ & 1.3 & 7.5 & $<0.001$ & 1.4 & 7.0 & $<0.001$ \\
\hline Malapposed struts, $\%$ & 0.5 & 1.1 & 0.51 & 0.5 & 0.9 & 0.58 \\
\hline Non-apposed side branch struts, $\%$ & 0.7 & 0.5 & 0.56 & 0.7 & 0.6 & 0.61 \\
\hline Neointimal coverage, $\mu \mathrm{m}$ & $85.5(61.9,124.1)$ & $69.5(32.7,127.5)$ & 0.20 & $82.7(60.5,118.8)$ & $76.6(36.2,139.5)$ & 0.84 \\
\hline
\end{tabular}

Data shown as numbers, percentages or median (IQR)

\section{Discussion}

The main finding of our study is that patients treated with BRS versus EES in the setting of AMI had significantly higher strut coverage as assessed by OCT at 6-8 month follow-up. Although neointimal maturity was comparable in both treatment groups in the overall cohort, in the sub-group of patients who presented with STEMI, it was significantly more advanced in BRS compared with EES. Neointimal area and thickness were both associated with subsequent TLF at 2 years post-implantation, irrespective of the implanted device. We found no association between neointimal maturity and subsequent TLF.

Apart from ISAR-Absorb MI [5], 10 other trials, namely ABSORB II [12], ABSORB III [13], ABSORB China
[14], ABSORB Japan [15], AIDA [16], EVERBIO II [17], TROFI II [4], ABSORB IV [18], COMPARE-ABSORB [19] and Seo et al. [20] have compared clinical outcomes after BRS or conventional metallic EES implantation. TROFI II was the only other randomized trial to exclusively include patients presenting with myocardial infarction. Patients who underwent BVS or EES implantation in the setting of STEMI underwent protocol specified 6-month angiographic and OCT follow-up [4], making it the most apt comparator of our study. As in the present study, the proportions of uncovered and malapposed struts in TROFI II were higher in the EES group as compared to the BRS group $(0.1 \pm 0.4$ vs. $0.0 \pm 0.1 \%, p=0.036)$ [4]. Specialized light property analysis of 6-month OCT pullbacks in the TROFI II cohort showed comparable light intensity, but lower light 
Fig. 3 Comparison of stent/ scaffold expansion indices between BRS and EES
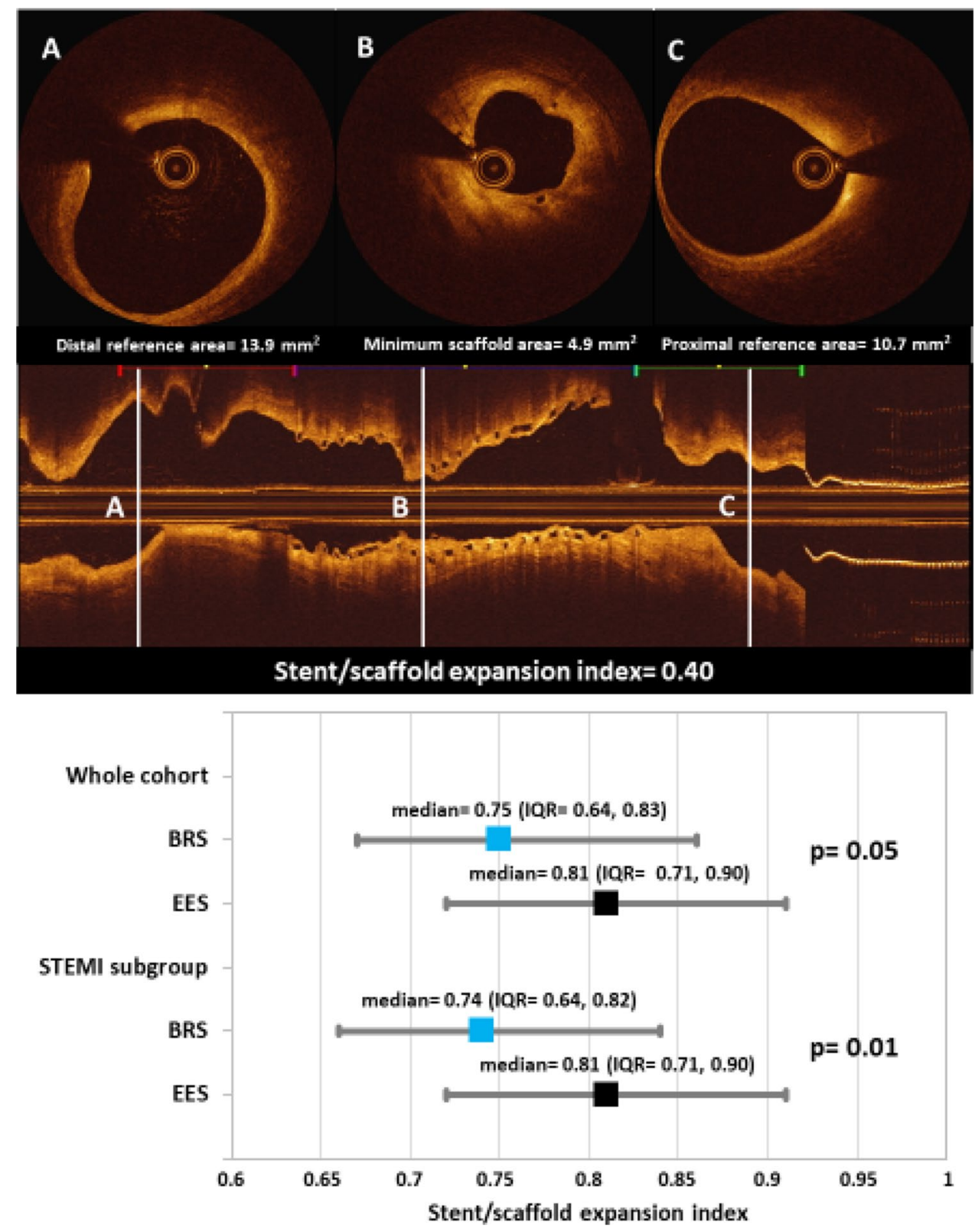

Table 5 Tissue characterization by grey-scale signal intensity analysis

\begin{tabular}{|c|c|c|c|c|c|c|}
\hline & \multicolumn{3}{|l|}{ Whole cohort } & \multicolumn{3}{|c|}{ STEMI subgroup } \\
\hline & BRS & EES & P-value & BRS & EES & $P$-value \\
\hline Patients, $\mathrm{n}$ & 65 & 30 & & 55 & 22 & \\
\hline Frames analyzed, $\mathrm{n}$ & 728 & 360 & & 558 & 276 & \\
\hline Regions of interest analyzed, $n$ & 2,233 & 1,210 & & 1,601 & 954 & \\
\hline Mean grey-scale signal intensity score & $\begin{array}{c}105.8(91.0 \\
121.0)\end{array}$ & $95.9(78.7,109.6)$ & 0.29 & $\begin{array}{c}104.8(90.6 \\
119.4)\end{array}$ & $\begin{array}{c}92.0(74.0, \\
107.4)\end{array}$ & 0.02 \\
\hline Mature regions of interest, $\%$ & 43.0 & 24.6 & 0.35 & 40.9 & 21.1 & 0.03 \\
\hline
\end{tabular}

Data shown as numbers, percentages or median (IQR) 
Fig. 4 Proportion of mature regions of interest in BRS and EES. Cut-off GSI score used for classification $=109.7$ (Malle et al., Arteriosclerosis, thrombosis, and vascular biology. 2013;33(6):1376-83.) [11]

\section{Grey-scale signal intensity analysis}
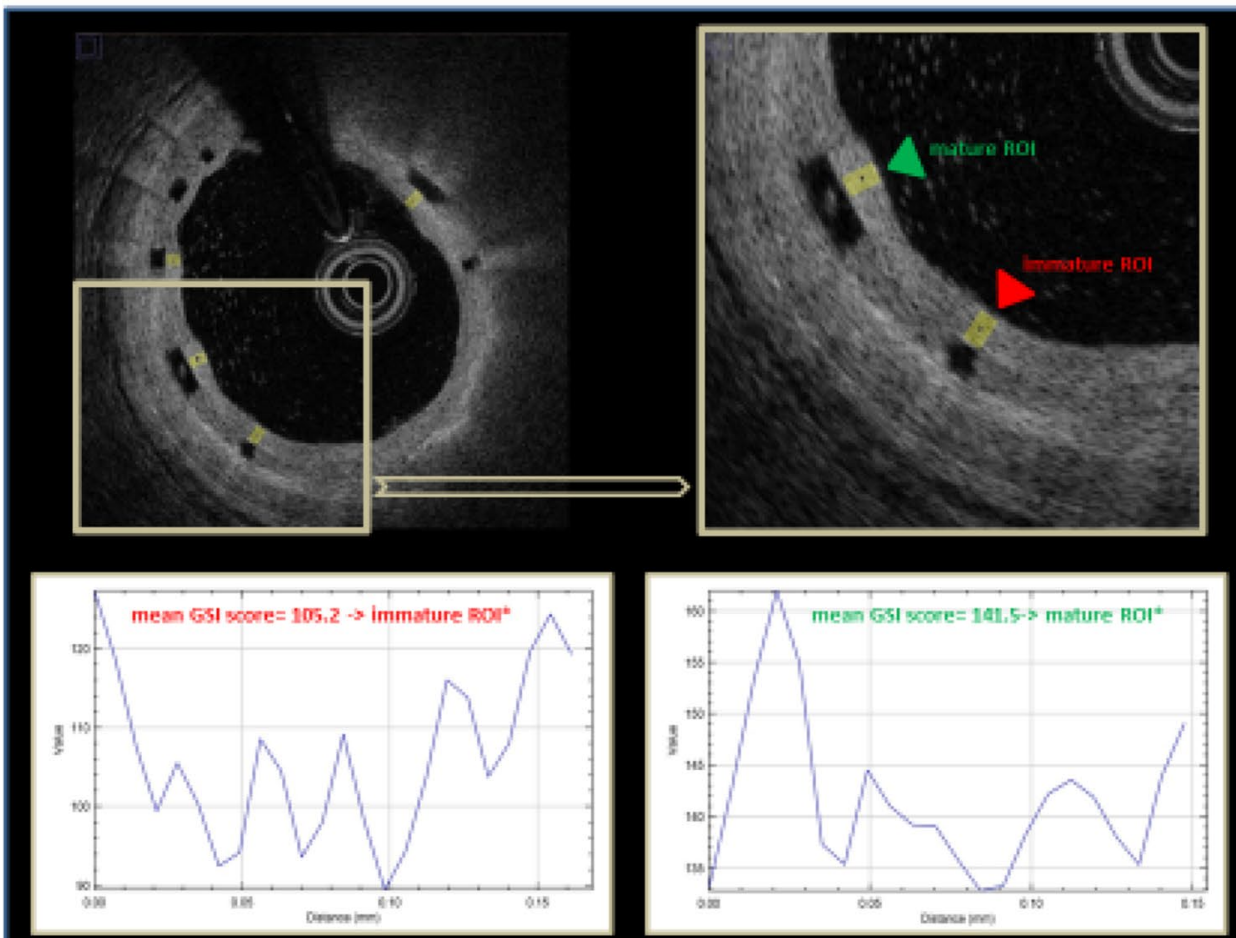

"Cut-off used for classification-109.7

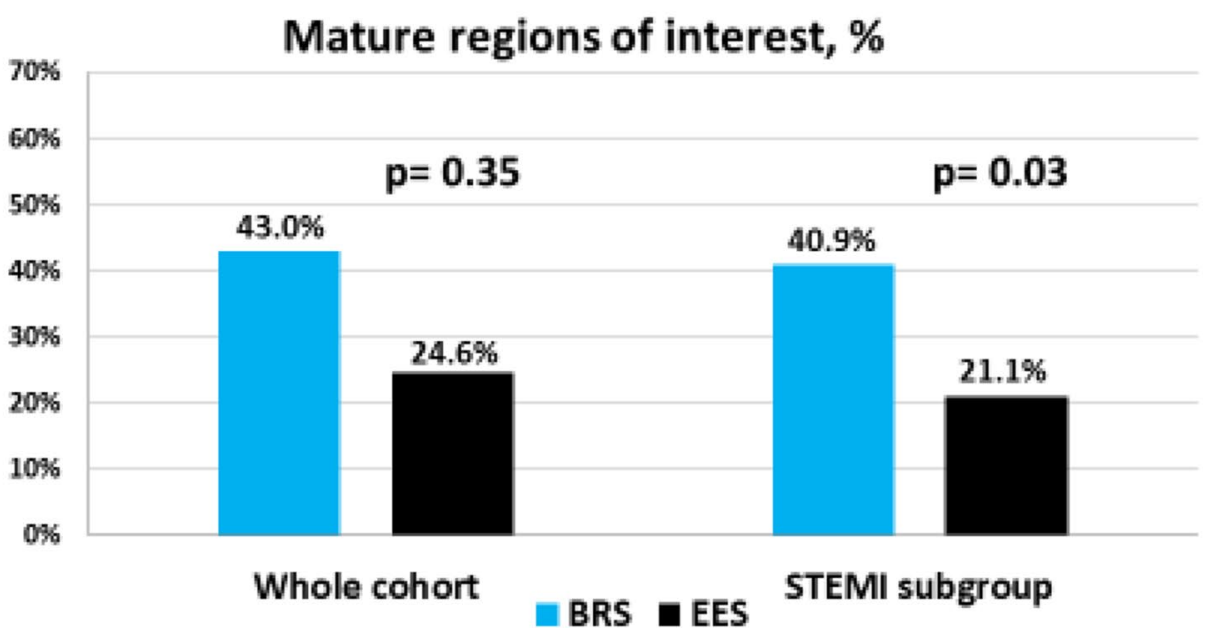

attenuation/ backscatter in superficial neointima in the BRS group as compared to EES [21]. This meant that BRS struts were enveloped by relatively stable superficial neointima with lower lipid components (lipid plaque, foam cells etc.) as compared to that in EES. Our GSI results are in line with the TROFI II neointimal findings, as we too report a numerically higher proportion of mature (homogeneous) neointimal ROIs in the BRS group as compared to EES, a difference that was statistically significant in our STEMI subgroup.

ABSORB BRS was officially discontinued in 2017 following concerns regarding higher rates of very late scaffold thrombosis (VLScT) compared with those observed in patients implanted with EES. Scaffold discontinuity, brought about by loss of structural integrity of BRS, which starts at around 12 months post implantation, has been identified as a leading cause of VLScT [22]. Discontinuous struts covered by thin-immature neointimal tissue comprised of fibrin or organized thrombus can dismantle and prolapse into the lumen; a process commonly known as late acquired malapposition. This exposes highly thrombogenic strut remnants to the blood, which has the potential to activate the coagulation cascade. On the other hand, mature neointimal tissue covering BRS struts should, in principle, play a critical role in long-term strut fixation. Near complete 

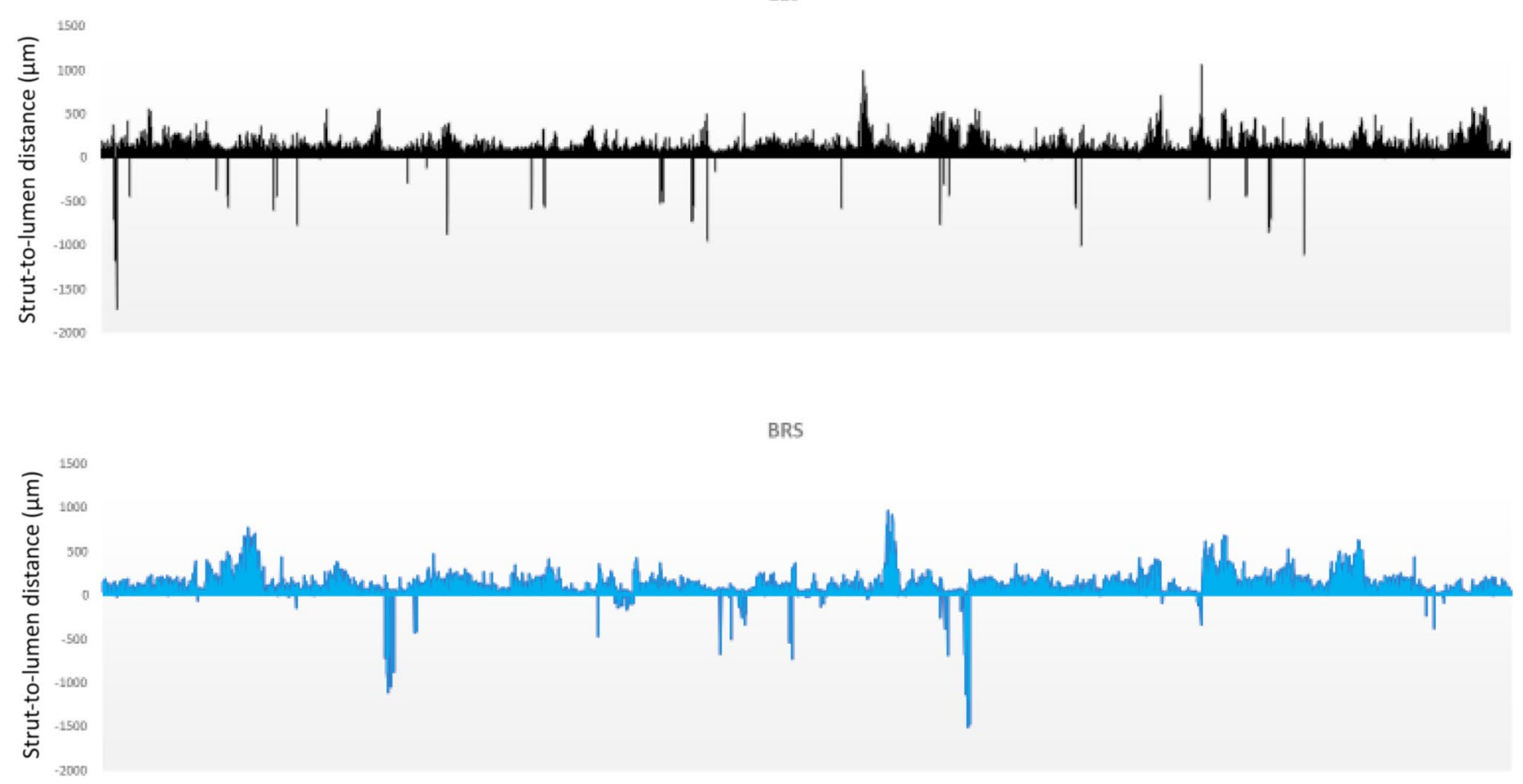

Fig. 5 Strut-to-lumen distances plotted for all analyzed struts. Strutto-lumen distance for each strut analyzed for all patients included in the analysis. Struts were considered covered if strut-to-lumen distance

reendothelialization of BRS was observed in our studied cohort at a relatively early phase (6-8 months post-implant) as evidenced by $>97 \%$ of strut coverage with $>42 \%$ mature neointimal ROIs. This left very few struts not yet covered with critical neointimal thickness. With the majority of struts already encapsulated by neointimal tissue at 6-8 month post implant time-point, future thrombotic events caused by scaffold discontinuity in this cohort seems unlikely.

BRS have been demonstrated to be non-inferior to EES in AMI settings [4, 5]. The majority of our cohort had STEMI as presentation diagnosis $(>82 \%)$. STEMI patients tend to be younger, with proximal soft lipid-rich lesions (with little or no calcification), which are often located in large caliber vessels. Such lesions seem attractive for BRS implantation. Restoration of a normal vessel physiology after complete resorption, brought about by normalization of vasomotion and compensatory remodeling have been observed in vessels implanted with BRS [23]. Implantation of bioresorbable scaffold instead of a permanent metallic stent in principle could be an ideal strategy in STEMI patients. Uncomplicated STEMIs could thus prove to be a potential niche for future iterations of fully resorbable scaffolds.

\section{Limitations}

Firstly, the present study involves a subset of cases from the ISAR-Absorb MI trial who underwent OCT at the time was $>20 \mu \mathrm{m}$ for EES and $>30 \mu \mathrm{m}$ for BRS. Negative strut-to-lumen distances indicated presence of malapposition

of surveillance angiography at the discretion of the operator. In such a scenario, selection-bias cannot be ruled out. Thus, some baseline characteristics between the two stent groups are slightly mismatched (EES cases were significantly older and had higher diameter stenosis at baseline). Secondly, since this was a post-hoc analysis on a subset of cases, the assessed sample size may not be enough to draw concrete conclusions, whilst still being hypothesis-generating. Thirdly, TLF assessment was done at 2 years post implant, while we know that scaffold resorption in Absorb BRS starts at around 12 months and completes at around 4 years post implantation. Irregular resorption process often leads to scaffold discontinuity which represents a primary cause of VLScT. Longer-term follow-up might be required to show a difference between the stent groups in terms of subsequent events. Fourthly, we cannot discount the fact that more aggressive lesion preparation and more frequent post-dilation used during BRS implantation caused a higher degree of vessel injury, which in turn would have contributed to the more robust healing response observed in BRS as compared to EES. This might have contributed to the observed lower rates of malapposition in patients treated with BRS. In addition, as systematic OCT imaging immediately post-procedure was not available for analysis, we are unable to comment on the evolution of stent expansion over time. Specifically, in the case of patients treated with BRS, it is possible that stent expansion reduced over time due to 
loss of radial strength of the scaffold, which might explain the constellation of lower rates of malapposition and lower stent expansion compared with EES.

\section{Conclusions}

In patients who underwent OCT surveillance 6-8 months after coronary intervention for AMI, with differing implantation characteristics depending on the device type used, vessel healing was more advanced in BRS compared with EES, particularly in the STEMI subgroup. Neointimal area and neointimal tissue thickness were the only OCT parameters associated with TLF at two years post-implantation.

Supplementary Information The online version contains supplementary material available at https://doi.org/10.1007/s10554-021-02251-x.

Funding Open Access funding provided by the IReL Consortium. No extramural funding was received for this analysis.

\section{Declarations}

Conflict of interest $\mathrm{RAB}$ reports research funding to the institution of prior employment from Celonova Biosciences. All other authors declare no conflicts of interest.

Open Access This article is licensed under a Creative Commons Attribution 4.0 International License, which permits use, sharing, adaptation, distribution and reproduction in any medium or format, as long as you give appropriate credit to the original author(s) and the source, provide a link to the Creative Commons licence, and indicate if changes were made. The images or other third party material in this article are included in the article's Creative Commons licence, unless indicated otherwise in a credit line to the material. If material is not included in the article's Creative Commons licence and your intended use is not permitted by statutory regulation or exceeds the permitted use, you will need to obtain permission directly from the copyright holder. To view a copy of this licence, visit http://creativecommons.org/licenses/by/4.0/.

\section{References}

1. Byrne RA, Stone GW, Ormiston J, Kastrati A (2017) Coronary balloon angioplasty, stents, and scaffolds. Lancet (London, England). 390(10096):781-92. https://doi.org/10.1016/S01406736(17)31927-X

2. Cassese S, Katagiri Y, Byrne RA, Brugaletta S, Alfonso F, Raber L et al (2020) Angiographic and clinical outcomes of STEMI patients treated with bioresorbable or metallic everolimus-eluting stents: a pooled analysis of individual patient data. EuroIntervention 15(16):1451-1457. https://doi.org/10.4244/EIJ-D-18-01080

3. Stone GW, Kimura T, Gao R, Kereiakes DJ, Ellis SG, Onuma Y et al (2019) Time-varying outcomes with the absorb bioresorbable vascular scaffold during 5-year follow-up: a systematic metaanalysis and individual patient data pooled study. JAMA Cardiol 4(12):1261-1269. https://doi.org/10.1001/jamacardio.2019.4101

4. Sabate M, Windecker S, Iniguez A, Okkels-Jensen L, Cequier A, Brugaletta S et al (2016) Everolimus-eluting bioresorbable stent vs. durable polymer everolimus-eluting metallic stent in patients with ST-segment elevation myocardial infarction: results of the randomized ABSORB ST-segment elevation myocardial infarction-TROFI II trial. Eur Heart J 37(3):229-240. https://doi.org/10. 1093/eurheartj/ehv500

5. Byrne RA, Alfonso F, Schneider S, Maeng M, Wiebe J, Kretov E et al (2019) Prospective, randomized trial of bioresorbable scaffolds vs. everolimus-eluting stents in patients undergoing coronary stenting for myocardial infarction: the Intracoronary Scaffold Assessment a Randomized evaluation of Absorb in Myocardial Infarction (ISAR-Absorb MI) trial. Eur Heart J 40(2):167-176. https://doi.org/10.1093/eurheartj/ehy710

6. Tearney GJ, Regar E, Akasaka T, Adriaenssens T, Barlis P, Bezerra HG et al (2012) Consensus standards for acquisition, measurement, and reporting of intravascular optical coherence tomography studies: a report from the International Working Group for Intravascular Optical Coherence Tomography Standardization and Validation. J Am Coll Cardiol 59(12):10581072. https://doi.org/10.1016/j.jacc.2011.09.079 (PubMed PMID: 22421299)

7. de Jaegere P, Mudra H, Figulla H, Almagor Y, Doucet S, Penn I et al (1998) Intravascular ultrasound-guided optimized stent deploymentXX Immediate and 6 months clinical and angiographic results from the Multicenter Ultrasound Stenting in Coronaries Study (MUSIC Study). Eur Heart J 19(8):1214-1223

8. Suwannasom P, Sotomi Y, Ishibashi Y, Cavalcante R, Albuquerque FN, Macaya $\mathrm{C}$ et al (2016) The impact of post-procedural asymmetry, expansion, and eccentricity of bioresorbable everolimus-eluting scaffold and metallic everolimus-eluting stent on clinical outcomes in the ABSORB II trial. JACC Cardiovasc Interv 9(12):1231-1242. https://doi.org/10.1016/j.jcin. 2016.03.027 (PubMed PMID: 27262861)

9. Koppara T, Tada T, Xhepa E, Kufner S, Byrne RA, Ibrahim T et al (2018) Randomised comparison of vascular response to biodegradable polymer sirolimus eluting and permanent polymer everolimus eluting stents: an optical coherence tomography study. Int J Cardiol 258:42-49. https://doi.org/10.1016/j.ijcard. 2018.01.011

10. Tada T, Kastrati A, Byrne RA, Schuster T, Cuni R, King LA et al (2014) Randomized comparison of biolimus-eluting stents with biodegradable polymer versus everolimus-eluting stents with permanent polymer coatings assessed by optical coherence tomography. Int J Cardiovasc Imaging 30(3):495-504. https://doi.org/10.1007/s10554-014-0376-1 (PubMed PMID: 24452729)

11. Malle C, Tada T, Steigerwald K, Ughi GJ, Schuster T, Nakano $M$ et al (2013) Tissue characterization after drug-eluting stent implantation using optical coherence tomography. Arterioscler Thromb Vasc Biol 33(6):1376-1383. https://doi.org/10.1161/ ATVBAHA.113.301227 (PubMed PMID: 23539216)

12. Serruys PW, Chevalier B, Sotomi Y, Cequier A, Carrie D, Piek JJ et al (2016) Comparison of an everolimus-eluting bioresorbable scaffold with an everolimus-eluting metallic stent for the treatment of coronary artery stenosis (ABSORB II): a 3 year, randomised, controlled, single-blind, multicentre clinical trial. Lancet 388(10059):2479-2491. https://doi.org/10.1016/S0140-6736(16) 32050-5 (PubMed PMID: 27806897)

13. Ellis SG, Kereiakes DJ, Metzger DC, Caputo RP, Rizik DG, Teirstein PS et al (2015) Everolimus-eluting bioresorbable scaffolds for coronary artery disease. N Engl J Med 373(20):19051915. https://doi.org/10.1056/NEJMoa1509038

14. Gao R, Yang Y, Han Y, Huo Y, Chen J, Yu B et al (2015) Bioresorbable vascular scaffolds versus metallic stents in patients with coronary artery disease: ABSORB China trial. J Am Coll Cardiol 66(21):2298-2309. https://doi.org/10.1016/j.jacc.2015.09.054 (PubMed PMID: 26471805) 
15. Kimura T, Kozuma K, Tanabe K, Nakamura S, Yamane M, Muramatsu $\mathrm{T}$ et al (2015) A randomized trial evaluating everolimuseluting Absorb bioresorbable scaffolds vs. everolimus-eluting metallic stents in patients with coronary artery disease: ABSORB Japan. Eur Heart J 36(47):3332-3342. https://doi.org/10.1093/ eurheartj/ehv435

16. Wykrzykowska JJ, Kraak RP, Hofma SH, van der Schaaf RJ, Arkenbout EK et al (2017) Bioresorbable scaffolds versus metallic stents in routine PCI. N Engl J Med 376(24):2319-2328. https:// doi.org/10.1056/NEJMoa1614954

17. Puricel S, Arroyo D, Corpataux N, Baeriswyl G, Lehmann S, Kallinikou Z et al (2015) Comparison of everolimus- and biolimus-eluting coronary stents with everolimus-eluting bioresorbable vascular scaffolds. J Am Coll Cardiol 65(8):791-801. https://doi. org/10.1016/j.jacc.2014.12.017

18. Stone GW, Ellis SG, Gori T, Metzger DC, Stein B, Erickson M et al (2018) Blinded outcomes and angina assessment of coronary bioresorbable scaffolds: 30-day and 1-year results from the ABSORB IV randomised trial. Lancet (London, England). 392(10157):1530-1540. https://doi.org/10.1016/S0140-6736(18) 32283-9

19. Smits PC, Genuns R (2018) COMPARE-ABSORB: 1-year results. TCT

20. Seo J, Ahn JM, Hong SJ, Kang DY, Hong SJ, Her AY et al (2020) Bioresorbable vascular scaffolds versus drug-eluting stents for diffuse long coronary narrowings. Am J Cardiol 125(11):1624-1630. https://doi.org/10.1016/j.amjcard.2020.02.031
21. Sotomi Y, Onuma Y, Liu S, Asano T, Eggermont J, Katagiri Y et al (2018) Quality difference of neointima following the implantation of everolimus-eluting bioresorbable scaffolds and metallic stents in patients with ST-elevation myocardial infarction: quantitative assessments by light intensity, light attenuation, and backscatter on optical coherence tomography in the TROFI II trial. EuroIntervention 14(6):678-685. https://doi.org/10.4244/ EIJ-D-17-00884

22. Yamaji K, Ueki Y, Souteyrand G, Daemen J, Wiebe J, Nef H et al (2017) Mechanisms of very late bioresorbable scaffold thrombosis: the INVEST registry. J Am Coll Cardiol 70(19):2330-2344. https://doi.org/10.1016/j.jacc.2017.09.014

23. Brugaletta S, Heo JH, Garcia-Garcia HM, Farooq V, van Geuns RJ, de Bruyne B et al (2012) Endothelial-dependent vasomotion in a coronary segment treated by ABSORB everolimus-eluting bioresorbable vascular scaffold system is related to plaque composition at the time of bioresorption of the polymer: indirect finding of vascular reparative therapy? Eur Heart J 33(11):1325-1333. https://doi.org/10.1093/eurheartj/ehr466

Publisher's Note Springer Nature remains neutral with regard to jurisdictional claims in published maps and institutional affiliations. 